\title{
Cross-cultural research must prioritize equitable collaboration
}

\author{
Research centres in low- and middle-income countries are routinely circumvented in the production of \\ cross-cultural research on human behaviour. Where local contributions are made, collaboration is rarely equitable \\ norms are overturned.

\section{Mark Urassa, David W. Lawson, Joyce Wamoyi, Eshetu Gurmu, Mhairi A. Gibson, Purnima Madhivanan and Caitlyn Placek} and often uncredited in co-authorship. Efforts to decolonize the social sciences will remain inadequate until these

$\mathrm{R}$ ecent months have witnessed a remarkable focus on racism and racial justice, highlighting an urgent need for greater respect of Black lives, along with global commitments to anti-racism. This shift has also had the welcome impact of reinvigorating efforts to decolonize the social sciences ${ }^{1}$. Numerous academic organizations and departments of anthropology, psychology, and related fields reliant on cross-cultural data production have now declared commitments to combat racism, diversify their syllabi, ensure ethical fieldwork practice, and improve representation of minoritized groups among their faculty and student body. Often missing from this discussion among high-income country (HIC)-based researchers, however, is the promotion of equitable collaboration in cross-cultural research with national universities and research centres in lowand middle-income countries (LMICs). While such centres sometimes have limited capacity, LMIC-based institutions and academics nevertheless exist and seek opportunities for intellectual engagement and access to funding streams (for example, refs. ${ }^{2,3}$ ). Furthermore, where LMIC researcher contributions are made in HIC-led projects, they are often deemed unworthy of authorship status, and payment for services is rarely channelled through institutional overheads capable of fostering national research infrastructure and career development ${ }^{4}$. These norms are extractive and woefully outdated, and until they are challenged and overturned, cross-cultural research on human behaviour will remain a robustly colonial enterprise.

Yet collaborative research in anthropology, cross-cultural psychology, and related social sciences is possible; the authors of this comment each represent cross-national research partnerships. Global health, a discipline with its own colonial trappings, is also increasingly taking a stand against 'parachute research' deemed parasitic to LMICs and communities supplying data that HIC scholars build their careers upon ${ }^{5-8}$. For example, several prominent global health journals now actively discourage manuscript submissions for which primary data has been collected in a LMIC without explicit collaboration and co-authorship with local researchers ${ }^{7}-$ a stance, to our knowledge, not shared by any social science journal, including Nature Human Behaviour. So, what are the barriers to equitable collaboration, why are less-applied forms of cross-cultural social science seemingly so stubbornly resistant to change, and what can we do about it?

\section{Barriers to equitable collaboration}

In our own experience, discussion of these issues with HIC-based colleagues brings up a number of barriers to equitable collaboration, at least some of which reflect misperceptions. Some claim that appropriate local expertise simply does not exist. This is of course a fantasy; few research projects in LMICs would be possible without tapping into local reservoirs of knowledge and expertise, which are indispensable in project design, data collection, interpretation and dissemination. Yet such contributions, be they from institutionally trained researchers, locally recruited fieldworkers, or community members, are often deemed inadequate to garner co-authorship by standards set by HIC researchers. This only poses the questions of whether the benchmarks of credited contribution are fairly constructed, why opportunities to be involved at 'sufficient' levels are often denied, and what improvements to such research practice could be enabled through greater involvement ${ }^{7}$.

We must also be cognizant of double standards. For instance, in return for piecemeal contributions to research, a colleague based in a HIC institution who conducts data analysis or analyses lab samples might rightfully anticipate co-authorship even without meaningfully contributing to study design or report writing. Why, then, are similarly vital contributions to field research, often spearheaded by collaborating LMIC researchers (for example, study tool refinement and implementation, translation work, facilitating local ethical approval, and liaising with community leaders and members while skilfully respecting local social obligations and norms) routinely disregarded when collaboration is acknowledged and made explicit via co-authorship?

Another argument is that certain projects require discipline-specific training sometimes lacking in LMICs. Differences in training may understandably stifle equitable collaboration in study design and, in extreme cases, potentially render co-authorship unethical if local contributors lack necessary skills to vouch for a study's conclusions. But here HIC researchers must be careful not to underestimate the wealth of expertise in contemporary LMICs, seek out appropriate expertise where it exists (including in neighbouring disciplines), and encourage local capacity building where it is absent. In this regard, long-term field projects in LMICs managed by HIC researchers that have run sometimes for decades without forming collaborative partnerships with national research centres and academics are especially disconcerting. Likewise, discussions of the ethics of cross-cultural research (for example, ref. ${ }^{9}$ ) that do not meaningfully incorporate the perspectives of LMIC-based researchers are likely to overlook issues of research capacity building and effective, ethical collaboration with LMIC institutions.

Others argue that researcher objectivity is obstructed by conflicts of interest between HIC and LMIC-based scholars. 
Anthropologists in particular often defend the benefits of an outsider view, a limiting stance if only HIC researchers get to occupy the privileged position of an outsider (see also ref. ${ }^{10}$ ). Yet, an outsider perspective also brings its own subjectivities, which can be counteracted through the co-creation of research with relative insiders to the culture or community under study ${ }^{6}$. Palpable conflict may arise because of differing worldviews and cultural values. There may be sociopolitical tensions between marginalized ethnic groups under study and national governments. There may also be differing research priorities. 'Blue sky' research questions about human behavioural variation, sometimes prioritized by HIC scholars, may appear trivial to LMIC researchers often more engaged with topics concerning human wellbeing. In this sense, we acknowledge, as authors of this comment, that our own connections to global health have laid clearer paths to collaborative partnerships. Each of these issues is complex and requires careful navigation. They are also not unsurmountable barriers and should be actively questioned when used to justify not pursuing collaborative relationships. As scholars of cultural diversity, we are up to the challenge of overcoming such differences in perspective. Change can come by shifting our priorities.

\section{Time for a norm change}

More than anything, the circumvention of LMIC researchers and national research infrastructure in the production of cross-cultural social science by HIC scholars is simply 'how business is done', an outdated norm driven by self-interest and due for revision. Research centres in HICs rest on a deep history of colonial interactions with LMIC institutions, researchers, and study communities that selectively support the careers and priorities of the former over the latter ${ }^{5}$. Challenging the status quo consequently comes with perceived sacrifices to a HIC-based agenda, researcher productivity, and career progression. This is evident at all levels.

In our experience, HIC graduate students in anthropology and cross-cultural psychology are typically encouraged to establish their own connections with study communities and are rarely advised to reach out to local research centres for assistance and collaboration. In job interviews, candidates advertise privileged access to 'their' field sites as assets to be added to the portfolios of HIC institutions. Sole and limited authorship are coveted. Pre-tenured faculty are discouraged from adding authors to their publications, so as not to minimize their own perceived contributions. Navigating collaborative partnerships takes time and effort, yet promotion committees demand evidence of near-constant productivity. Principal investigators are reluctant to channel limited research funding through LMIC research centres if it can be avoided. Funding agencies routinely define 'broader impacts' only in terms of domestic interest, failing to reward projects that boost research capacity in LMICs. HIC institutions hosting grants demand high overheads and often restrict opportunities to pay overheads to overseas institutions. In some contexts, HIC researchers ignore national ethical review boards altogether. In others, indigenous researcher concerns over the discontent of 'research-fatigued' study communities are disregarded in the face of HIC demands for rapid, low-cost data production $^{11}$. Finally, even well-intentioned early-career HIC researchers can be hesitant to adopt more equitable practices for fear of 'rocking the boat' in field projects wherein leadership positions are occupied by powerful senior colleagues.

At each of these levels, we must now hold one another accountable to change. Addressing structural barriers within universities, grant-funding bodies, journals, and academic societies presents a fundamental challenge. HIC institutions do not always value capacity building, mentorship, or the time taken to build partnerships in evaluation and promotion procedures. Increasingly, some funders welcome, or even require, that data collection in LMICs is accompanied by financial support of LMIC institutions (for example, the Wellcome Trust and the Global Challenges Research Fund in the United Kingdom), but this remains more typical of global health than anthropology or psychology. Beyond financial support, leadership must be devolved to recognize the authority and jurisdiction of LMIC institutions. This must include seeking out and supporting LMIC ethics panels, editors, and grant reviewers, as well as prioritizing national legislation and financial accounting systems. Critically, while institutional barriers may be slow to dismantle, as individual scholars based in both HICs and LMICs we must recognize and take immediate opportunities to facilitate academic capacity-building and promote equitable collaboration (Table 1 ). Individual action is a fundamental and necessary step in promoting institutional transformation.

These issues are particularly relevant in the light of a recent awakening among social scientists to the value of cross-cultural data $^{9}$. As HIC researchers expand sampling of 'diverse' populations (especially among people of colour ${ }^{12}$ ), it is time to adopt equal enthusiasm for shaking off extractive practices. Critical here is a realization that meaningful change cannot be achieved simply via adopting the present standards of ethical review boards, which are often focused solely on risk minimization to study communities and researchers, while overlooking the commodification of field research and the equitable share of its rewards. Nor is the economic compensation of localized study participants sufficient. Paying for an individual's time to conduct a survey, paying for schoolbooks or medical care, or offering aid in times of shortfall, are all important and beneficial activities characteristic of many long-term HIC-led field programs. Participatory research methods can also ensure community involvement and effective research dissemination at a local scale 9 . But, let us be clear that such activities rarely build national academic capacity and research infrastructure, which are too often left dependent on external expertise with limited opportunity for independent growth.

\section{The way forward}

Authorship, and access to it, is a discernible indicator of LMIC scholar involvement and can provide required opportunities for professional networking, career progression, and academic independence ${ }^{6}$. For this reason, we have highlighted issues of authorship. However, we must also avoid 'token' assignment of LMIC authors to manuscripts in place of growing truly equitable partnerships defined by genuine intellectual collaboration. Inundating LMIC researchers with requests to collaborate will be futile and counterproductive if requests are not both backed financially and approached flexibility. HIC researchers must avoid pressuring LMICs to collaborate and co-author simply to give the appearance of equitable partnerships. Instead we must commit to truly transformative change via our institutions and individual actions.

In closing, we emphasize that the ultimate goal is not merely to more fully recognize LMIC researchers as collaborative partners, but to enable equal opportunity for leadership rather than simply participation in cross-cultural social science. Building equitable collaborations offers not only immediate benefits to the careers and livelihoods of LMIC scholars, but also critical opportunities to foster independent research infrastructure. We must be realistic that true independence will take time to establish and will require investment and initiative from both HICs and LMICs. In many cases, LMIC research institutions were 
Table 1 | Practical recommendations for fostering equitable collaboration in cross-cultural social science

All researchers

- Adopt a flexible research agenda, exploring shared priorities with (potential) collaborative partners.

- Campaign funders to enable equitable grant sharing between HIC and LMIC institutions.

- Designate co-authorship where credit is due, applying equal expectations to HIC and LMIC collaborators.

- Embrace open-access publishing and data sharing to ensure global access to research.

- Decolonize and diversify syllabi, acknowledging inequities in cross-cultural research norms and the urgent need for change.

- Strengthen teaching of research ethics into curriculum, including dangers of extractive research.

- Embrace opportunities (for example, social media) to advertise your expertise to a global audience and encourage collaborative opportunities.

- Develop and enforce ethical review procedures defining and encouraging equitable collaboration between LMIC and HIC institutions and researchers.

- Support LMIC-based journals and academic societies, operating independently of HIC agendas. Hold 'international' society meetings in LMICs and/or virtually.

- Develop mentoring arrangements between HIC and LMIC researchers (for example, assistance with project design, funding applications, English language proof-reading, and navigating the publication ecosystem), including via initiatives such as AuthorAid (https://www.authoraid.info/en/).

HIC researchers - Increase awareness of LMIC institutions and scholars, and seek out local collaborative partners.

- Where possible, factor LMIC institutional overheads and capacity building activities into research from the outset.

- Avoid 'token' LMIC co-authorship. Strive for genuine intellectual collaboration and financially supported partnerships.

- Diversify HIC-based faculty and student bodies, journal editors, and funding agency program officers via recruitment activities.

- When visiting LMICs (for example, to collect data), offer to provide training at LMIC universities and research centres.

- Share unused field equipment with LMIC partners after or during dormant periods of data collection.

- Avoid possessive references to study communities (for example, 'my field site'), which can falsely imply ownership of access to a community.

- Reward the time and effort required to forge equitable collaborations as a reviewer in recruitment, promotion, and funding allocation decisions.

LMIC researchers - Negotiate authorship inclusion and position, along with data sharing, in collaborative research with HICs. When possible, reject proposals for collaboration which are not equitable.

- Support appropriate, transparent budgeting within LMIC institutions to facilitate access to international grants and reduce dependence on HICs.

- Strengthen teaching in the social sciences, especially beyond global health. Foster positive aspirations, knowledge and access to opportunities for early-career LMIC researchers.

- Increase LMIC scholar visibility by negotiating to present collaborative and independent research at international conferences.

- Host HIC students and study abroad programs, which can be profitable and increase LMIC institution visibility.

- Strengthen collaborative research networks with HICs, especially with researchers originating from LMICs to counteract effects of 'brain drain'.

- Take advantage of publication fee waivers for LMIC authors in journals where they exist.

- Communicate needs and priorities. HIC scholars may be eager to collaborate, but are rarely accustomed to different realities or expectations of LMIC research centres. Guide good intentions to effective actions.

Note: The HIC-LMIC division serves as practical heuristic to highlight the need for somewhat distinct priorities by context. We recognize that it necessarily simplifies the diversity of contexts under which research is conducted. Our recommendations are not exhaustive and are based primarily on our experiences in anthropology and global health, with the intention to stimulate further discussion.

initiated by HIC resources and continue to be highly dependent on external funding in the face of limited national research budgets $^{3}$. The strengthening of LMIC institutional research capacity is thus a fundamental priority.
We also stress the scientific case for equitable collaboration. It is now well-established that diverse and inclusive research teams stimulate innovation and achieve greater scientific impact ${ }^{13}$. Local partnerships also offer the benefits of a more adaptive global research ecosystem, capable of responding to unexpected change. As has recently been highlighted in biology ${ }^{14}$, HIC-led projects that invested early in LMIC research partners are now more able to weather the storm caused by the COVID-19 pandemic and ensuing travel restrictions. Social science projects that rely on regular data collection among LMIC populations are also currently facing an uncertain future as fieldwork is cancelled or postponed indefinitely. So, let's harness this unexpected opportunity for reflection and the renewed energy of decolonizing efforts and commit to a future in which equitable collaboration is no longer remarkable, but rather the norm in cross-cultural social science.

\section{Mark Urassa (D) 1 四, David W. Lawson (D) 2 幽, Joyce Wamoyi (iD), Eshetu Gurmu3 ${ }^{3}$, Mhairi A. Gibson (D) \\ Purnima Madhivanan (DD) 5,6 and Caitlyn Placek ${ }^{7}$ ${ }^{1}$ National Institute for Medical Research, Mwanza, Tanzania. ${ }^{2}$ Department of Anthropology, University of California Santa Barbara, Santa Barbara, CA, USA. ${ }^{3}$ Centre for Population Studies and Institute of Development and Policy Research, Addis Ababa University, Addis Ababa, Ethiopia. ${ }^{4}$ Department of Anthropology and Archaeology, University of Bristol, Bristol, UK. ${ }^{5}$ Public Health Research Institute of India, Mysore, India. ${ }^{6}$ Department of Health Promotion Sciences, Mel \& Enid Zuckerman College of Public Health, University of Arizona, Tucson, AZ, USA. ${ }^{7}$ Department of Anthropology, Ball State University, Muncie, IN, USA.

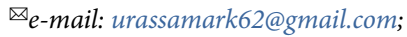 dlawson@anth.ucsb.edu}

Published online: 18 March 2021

https://doi.org/10.1038/s41562-021-01076-X

\section{References}

1. Smith, L.T. Decolonizing Methodologies: Research and Indigenous Peoples. (Zed Books, 2013).

2. Fussy, D. S. High. Educ. 77, 283-299 (2019).

3. Magesa, S.M., Mwape, B. \& Mboera, L.E.G. Tanzan. J. Health Res. https://doi.org/10.4314/thrb.v13i5.11 (2011)

4. Wight, D. Soc. Sci. Med. 66, 110-116 (2008).

5. Boum, Y. et al. BMJ Glob. Health 3, e000868 (2018).

6. Abimbola, S. BMJ Glob. Health 4, e002068 (2019).

7. Lancet Glob. Health 6, e593 (2018).

8. Jumbam, D. T. BMJ Glob. Health 5, e003164 (2020).

9. Broesch, T. et al. Proc. R. Soc. Lond. B 287, 20201245 (2020).

10. Nordling, L. Who gets to study whom? Sapiens https://www sapiens.org/culture/anthropology-colonial-history/ (17 July 2020).

11. Kalinga, C. J. Afr. Cult. Stud. 31, 270-272 (2019).

12. Clancy, K. B. H. \& Davis, J. L. Annu. Rev. Anthropol. 48, 169-186 (2019).

13. AlShebli, B. K., Rahwan, T. \& Woon, W. L. Nat. Commun. 9 , 5163 (2018).

14. de Vos, A. The problem of 'colonial science'. Scientific American https://www.scientificamerican.com/article/ the-problem-of-colonial-science/ (1 July 2020). 
Acknowledgements

M.U. and D.W.L. contributed equally and share first

authorship. D.W.L. is supported by the National Science

Foundation (award number: 1851317). M.A.G. and E.G.

are supported by the British Academy (award number:

HDV190133). P.M. is partially supported by the National
Institutes for Health Fogarty International Center (award number: D43 TW010540).

Competing interests

The authors declare no competing interests.
Additional information

Peer review information Nature Human Behaviour

thanks Johnson Muchunguzi Ishengoma,

Ndukuyakhe Ndlovu, Andy Nobes, and Rebecca

Sear for their contribution to the peer review of this work. 